\title{
Das Bunker-Hill-Projekt in Los Angeles
}

Lärm und Schmutz, soziale und rassenbedingte Spannungen, sowie wachsende Kriminalität waren während Jahrzehnten die Hauptursachen für die Abwanderung der materiell besser gestellten weissen Mittelschicht aus den amerikanischen Innenstädten in die Vorortsgemeinden. Begünstigt wurde dieser Exodus durch die starke Zunahme des motorisierten Privatverkehrs, durch den Bau von Schnellverkehrsstrassen und durch die Förderung des Eigenheimbaus durch die Regierung.

Schwächere Einkommensgruppen, vor allem Schwarze und Latinos, füllten den durch den Auszug der kapitalkräftigeren Bevölkerung freigewordenen Raum aus. Für die Haus- und Grundbesitzer hatte diese Umschichtung einen empfindlichen Rückgang der Mieteinnahmen und einen beträchtlichen Kapitalverlust durch das Fallen der Bodenpreise zur Folge. Kein Wunder, dass der Gebäudeunterhalt immer mehr vernachlässigt wurde, was zu eimen Zerfall der Wohnsubstanz und damit zu einer Verslumung der betreffenden Quartiere führte.

In Downtown Los Angeles zeigte sich dieser unaufhaltsame Zerfall der menschenwürdigen Lebensbedingungen am deutlichsten im Raum von Bunker Hill. Bereits in den fünfziger Jahren erklärte das "City Department of Building and Safety" 60 : der 395 Gebäude in Bunker Hill als abbruchreif, und nach Ansicht des "City Health Departments" genügten von den 4'800 Wohnungen nur noch 18 \% den minimalsten hygienischen und sanitären Anforderungen. Der Polizei bereitete das Bunker Hill Quartier ebenfalls immer grössere Srrgen, denn die Zahl der Verhaftungen lag hier 1958 767 : über dem städtischen Durchschnitt. 'Villiam T. Sesnon Jr., der erste Vorsitzende der "Community Redevelopment Agency" (CRA) sagte im gleichen Jahr vor dem City Council unter anderem: "It will be interesting to you as Councilmen to know that the total cost of certain health, fire and police services alone in Bunker Hill for the past fiscal year was 754'101 dollars while the revenue was 94'210 dollars. It should be borne in mind that the substantial difference between property tax revenue and costs of city services must be borne by the taxpayers of othe $r$ areas in the city."

Aber nicht nur die Einnahmen der Stadt gingen zurück, sondern auch die Umsätze der Warenhäuser, der Detailgeschäfte, der Hotels usw. Neben dem baulichen machte sich somit auch ein wirtschaftlicher Niedergang immer deutlicher bemerkbar. Massnahmen zur Rettung des Bunker Hill Ge- bietes mussten daher dringend eingeleitet 'verden. Schon im März 1959 hiess deshalb der City Council das vorgelegte Sanierungsprogranm gut, und die "Community Redevelopment Agency" erwarb daraufhin im Gebiet zwischen der First Street im Norden, der Hill Street im Osten, der Fifth Street im Süden und dem Harbor Freeway im Westen 285 Grundstücke. Die darauf lebenden 5'617 Personen wurden ungesiedelt, und für die 442 vorhandenen Geschäfte suchte man neue Standorte. Dann begann man mit dem Abbruch von 393 baufälligen Häusern. Zum grössten Teil handelte es sich dabei um drei- bis viergeschossige Holzbauten ohne Aufzüge, sogenannte walk-ups. Anschliessend wurden die auf dem freigelegten Areal bestehenden grösseren Gefällsunterschiede durch massive Abtragungs- und Aufschüttungsarbeiten weitgehend ausgeglichen.

Die diesem Artikel beigegebene Luftaufnahme zeigt einen Ausschnitt des Downtown Gebietes von Los Angeles von Norden her. Im Vordergrund führt der Hollywood Freeway über den Harbor Freeway mit seirien verschiedenen Aus- und Zufahrtsstrassen hinweg. Das Gebäude oberhalb der Bildmitte links, mit den konvexen Längsseiten, ist ein $\mathrm{Pa}$ villon des Music Centers. Ihm gegenüber, fast doppelt so hoch, erhebt sich der wuchtige Block des Water and Power Buildings. Beide Gebäulichkeiten gehören zum Civic Center, von dem aber hier nur ein kleiner Teil zu sehen ist. Die Strasse, die den Schmalseiten der beiden Bauwerke entlang, mehr oder weniger quer durch die obere Bildhälfte verläuft, ist die First Street. Von dieser weg bis zum völlig überbauten Gebiet am oberen Bildrand erstreckt sich das 136 acres - etwa 55 Hektaren - umfassende Bunker Hill Areal.

Bis Ende der sechziger Jahre, als diese Aufnahme gemacht wurde, konnten von den geplanten Bauvorhaben erst deren zwei verwirklicht werden. Nämlich der 42 Stockwerke hohe, schlanke Wolkenkratzer der Union Bank (in der rechten oberen Bildecke) und die drei Wohnblöcke, die Bunker Hill Towers (in der Mitte der freien Fläche der oberen Bildhälfte). Letztere enthalten zusammen 725 Appartements.

Heute bietet sich dem Downtown-Besucher bereits ein wesentlich anderer Anblick. Vor dem Zwillingswolkenkratzer am oberen Bildrand steht das mit einem Kostenaufwand von 110 Millionen Dollar er-

Prof. Dr. Heinrich Inhelder, Geographielehrer, Buchzelgstrasse 32, 8053 Zürich 
richtete Bonaventure Hotel. Ein phantastischer Bau, der in fünf mit Spiegelglas verkleideten, zylindrischen Türmen über 1'500 Gästezimmer verfügt und auf acht Ebenen, rings um ein Atrium angeordnet, zahlreiche Ladengeschäfte, Restaurants und Bars beherbergt. In der Blickrichtung links neben diesem ultramodernen Hotel befindet sich jetzt die Atlantic Richfield Plaza Garage und zwischen dem Bonaventure Hotel und dem Komplex der Bunker Hill Towers steht das Los Angeles World Trade Center. Vor der Atlantic Richfield Plaza Garage ragt neuerdings das höchste Gebäude der Ueberbauung, der 55 Etagen zählende Wolkenkratzer der Security National Pacific Bank zum kalifornischen Hinmel empor. Die niedrig gehaltene Central Plant zu seinen Füssen verschwindet daneben fast ganz.

Das Bautempo und die Investitionsfreudigkeit privater Kreise nahm in den vergangenen Jahren stark $z u$, denn immer deutlicher begannen sich lohnende wirtschaftliche Möglichkeiten in Bunker Hill abzuzeichnen, was vereinzelte Firmen veranlasste, ihren Standort aus den Vororten ins Stadtzentrum zu verlegen. Andere dagegen betrachten die Entwicklung noch etwas misstrauisch und nehmen weiterhin eine abwartende Haltung ein. Die Reaktivierung der Innenstadt ist aber in vollem Gange, und das Ziel, die Verwirklichung eines lebensfähigen Mehrzweckbezirkes, rückt in greifbare Nähe. Bis Mitte der achtziger Jahre ist der Bau weiterer Wohneinheiten für untere und mittlere Einkommen, sowie eine Senior Citizen Siedlung mit allen notwendigen Einrichtungen für eine medizinische und fürsorgerische Betreuung der Betagten vorgesehen. Ferner sind weitere $B a$ ten für Büros, Detailgeschäfte, Hotels, Theater und Kinos geplant. Die lockere Bauweise lässt z'ddem überall genügend Platz für Grünflächen, Blumenbeete, Erholungsräume und für Freizeiteinr' zhtungen wie zum Beispiel Tennisplätze und Swimmingpools. Nach Vollendung des Projektes werden ungefähr 7'000 Menschen in Bunker Hill wohnen und rund 60'000 werden hier täglich ihre Arbeitsplätze aufsuchen.
Die Verkehrsplanung sah von Anfang an für das ganze Bunker Hill Areal eine strikte Trennung des Fussgänger- und Fahrzeugverkehrs vor. Ein spezielles Pedway-System sorgt deshalb heute dafür, dass Angestellte, Passanten, kauflustige Besucher und Touristen sicher und möglichst bequem jeden Punkt innerhalb der überbauten Zone erreichen können. Ueberdachte Treppenaufgänge, Lifts und Rolltreppen bringen jedermann auf eine über dem öffentlichen Verkehr liegende Fussgängerebene mit geräumigen Plätzen und Promenaden. Brücken erlauben das gefahrlose Ueberqueren der starkbefahrenen Durchgangsstrassen. Für einen schnellen und reibungslosen Massentransport innerhalb des Bunker Hill Distriktes und zur Aufrechterbialtung der Verbindungen zu den umgebenden Quartieren ist ein "People Mover" vorgesehen, in der Art des Monorails in Disney World in Florida. Man hofft, damit auch den privaten Autoverkehr auf ein vernünftiges Mass eindämmen żu können. Bis zur Verwirklichung dieses Projektes übernehmen vorderhand Minibusse diese Aufgabe.

Nach Ansicht der Verantwortlichen entsteht in Bunker Hill eine Art "Vorstadt in der Innenstadt", eine Lebensgemeinschaft, die nicht nach Büro- und Ladenschluss zerfällt und verödet. Gilbert Linsday, ein Mitglied des City Council, ist der Auffassung, dass Bunker Hill in zehn Jahren zusammen mit dem Civic Center die schönste und grossartigste Downtown in ganz Amerika sein wird. Umfragen haben jetzt schon deutlich gezeigt, dass viele Einwohner der Suburbs aus den verschiedensten Gründen bereit wären, in einen solchermassen sanierten und attraktiven Stadtkern zurückzukehren. Viele fühlen sich schon seit langem in den Vororstgemeinden verlassen und vermissen die Möglichkeit, am kulturellen Leben teilzunehmen oder einfach allein oder mit Freunden ausgehen zu können. Auch die Aussicht, den Arbeitsplatz oder die Einkaufsgeschäfte zu Fuss zu erreichen, ist für viele verlockend. Eine Wende, die in andern amerikanischen Städten ebenfalls zu beobachten ist, bahnt sich hier in Los Angeles an.

\section{Literatur}

Boesch, Hans: USA, Werden und Wandel eires kontinentalen Wirtschaftsraumes. Künmierly \& Frey, Geographischer Verlag Bern, 1973.

Harms: Handbuch der Geographie. Band Amerika, Paul List Verlag München, 1975.
Der Spiegel: Zurück in die City. Nr. 25, 19.Juni 1978.

Bunker Hill Completion Programm 1977 - 1986. The Community Redevelopment Agency of the City of Los Angeles, California, 1977. 
